Commun. math. Phys. 5, 23-41 (1967)

\title{
Mixture of Outgoing and Incoming Electromagnetic Radiation. Change of Mass of the Source of Radiation*
}

\author{
M. A. Rotenberg, Ph.D. \\ The Negev Institute for Arid Zone Research, Beersheva, Israel
}

Received January 11, 1967

\begin{abstract}
In a previous paper the author, using a method of successive approximations, verified by means of the Einstein-Maxwell equations of general relativity the well-known result that outgoing electromagnetic radiation from a source conveys energy, so that the source loses gravitational mass corresponding to this energy. The purpose of this work is to show a similar result for the general case of any mixture of outgoing and incoming radiation.
\end{abstract}

\section{Introduction}

From everyday use of electromagnetic waves it is evident that they carry energy. Any source that emits them must lose energy. In a previous paper (ROTENBERG, 1966) this was verified via a method of approximation applied to the Einstein-Maxwell equations

$$
R_{i k}=-8 \pi E_{i k}
$$

for free space, by investigating radiation from a simple source - an electric dipole oscillating smoothly for a finite period. It was shown that the source suffers a permanent reduction of gravitational mass equal to the total energy of radiation emitted. The result referred to outgoing waves only; the present work sets out to show a similar result for any mixture of outgoing and incoming waves, having as the source (and receiver) the electric dipole just mentioned.

The electric dipole is explained more clearly in section 2 , and in sections 3 and 4 the metric and method of approximation are described. The solution (obtained in section 5 for the dipole) of the wave equation for the electromagnetic 4-potential is needed in section 6 to calculate the electromagnetic energy tensor and the total flux of energy of electromagnetic waves from the source. Finally, the main result, that the source undergoes a secular variation in mass equal and opposite to the total flux of energy of electromagnetic radiation, is established in section 7. The more complicated calculations occur in two appendices, followed by a third appendix containing a notation connected with mixed, outgoing and incoming, radiation and used frequently in this paper.

* This work is included in a thesis submitted by the author (1964) to the University of London for the degree of Ph.D. 


\section{The Source}

We shall consider an electric dipole which consists of two particles $A$ and $B$ of equal, negligible mass $\frac{1}{2} m$ and carrying equal and opposite charges $\pm e$; the particles are made to vibrate symmetrically along the axis $O z$ (of a rectangular Cartesian coordinate system) about their midpoint $O$, the origin. Let us specify the positions of $A$ and $B$ at time $t$ by

$$
A=[0,0, \zeta(t)], \quad B=[0,0,-\zeta(t)],
$$

where $\zeta(t)$ is any given bounded function of $t$ which is (i) constant outside the finite interval $t_{1} \leqq t \leqq t_{2}$, (ii) single valued and has unique derivatives of all orders in the interval $t_{1} \leqq t \leqq t_{2}$ : thus the system is supposed to vibrate smoothly and only between times $t_{1}$ and $t_{2}$. We assume that the arbitrary motion of the charged particles, described by $\zeta(t)$, is caused by a mechanical device (such as a spring) of negligible mass, insulated from the charges (so that $e=$ const.) and confined within a finite region near $O$. Uniform motion of the charges is ruled out.

Finally, the oscillation of the two particles of the dipole may be regarded as partly the cause of the outgoing waves and partly the effect of the incoming waves: thus the system acts as a 'source and receiver' of the waves.

\section{The Metric}

For the source introduced in section 2 (which is axially symmetric about $O z$ ) we shall, following Bonnor (1959), employ the axi-symmetric metric

$$
d s^{2}=-A d r^{2}-r^{2}\left(B d \theta^{2}+\sin ^{2} \theta C d \phi^{2}\right)+D d t^{2}:
$$

$(r, \theta, \phi)$ are the spherical polar coordinates of the field-point $P$ and $A, B, C, D$ are functions of $r, \theta, t$. This metric is the diagonalized form of the more general axi-symmetric metric

$$
\begin{aligned}
d s^{2}=-A d r^{2}- & r^{2}\left(B d \theta^{2}+\sin ^{2} \theta C d \phi^{2}\right)+ \\
& +D d t^{2}+2 r E d r d \theta+2 F d r d t+2 r G d \theta d t,
\end{aligned}
$$

$E, F, G$ also being functions of $r, \theta, t$.

\section{The Method of Approximation}

In section 6 , the formula for the electromagnetic energy tensor $E_{i k}$ for the dipole of section 2 turns out to be a double-parameter expansion of the form

$$
E_{i k}=\sum_{p=2}^{\infty} \sum_{s=2}^{\infty} e^{p} a^{s} E_{i k}^{(p s)}\left(x_{l}\right)
$$

in terms of the constants $e$ and $a$. The parameter $e$ is the charge of the particle $A$ of the dipole as in section 2 ; the parameter $a$, having the 
dimensions of length, is defined by

$$
\zeta(t)=a f(t)
$$

where $\zeta(t)$ is given by equations (2.1) and $f(t)$ is dimensionless. We shall, therefore, suppose that the metric tensor can similarly be expanded:

$$
g_{i k}=\stackrel{(00)}{g}_{i k}+\sum_{p=2}^{\infty} \sum_{s=2}^{\infty} e^{p} a^{s} g_{i k}^{(p s)}\left(x_{l}\right)
$$

due consideration being given to the fact that $\stackrel{(00)}{g}_{i k}$ refers to flat spacetime.

The method of approximation to be employed here involves the expansions (4.1) and (4.3) for the $E_{i k}$ and $g_{i k}$. Inserting these expansions in the field equations (1.1) and equating the coefficients of $e^{p} a^{s}$, we get what is to be called the $(p s)$ approximation, namely, a set of secondorder differential equations of the form

$$
\Phi_{l m}\left(\stackrel{(p s)}{g}_{i k}\right)=\stackrel{(p s)}{\Psi}_{l m}\left(\stackrel{(q r)}{g}_{i k}\right)+\text { const. } \times \stackrel{(p s)}{E}_{i k} .
$$

In these, the $\Phi_{l m}$ are linear in $\stackrel{(p s)}{g}_{i k}$ (and their derivatives); the $\stackrel{(p s)}{\Psi}_{l m}$ are nonlinear in $\stackrel{(q r)}{g}{ }_{i k}(q \leqq p-2, r \leqq s-2)$ (and their derivatives) known from previous approximations. Thus, apart from the expressions in $\stackrel{(22)}{E}_{i k}$, the (22) approximation contains only terms linear in $\stackrel{(22)}{g}_{i k}$ (and their derivatives); the nonlinear expressions $\stackrel{(p s)}{\Psi}_{l m}$ in equations (4.4) do not appear in the approximation. It is in the solution of this (22) approximation that there first appears a term representing a permanent change of gravitational mass of the source equal and opposite to the total flow of energy of electromagnetic waves from the source (section 7). So our aim is to get an appropriate solution of the (22) approximation, which is achieved in section 7 .

The solution of the $(p s)$ approximation, the $(p s)$ solution, is the $\stackrel{(p s)}{g}_{i k}$ which satisfy equations (4.4).

To conclude this section we form expansions for the coefficients of the metric (3.1): in virtue of equations (4.3) they are

$$
\begin{aligned}
-g_{11}=A & =1+\sum_{p=2}^{\infty} \sum_{s=2}^{\infty} e^{p} a^{s} \stackrel{(p s)}{A}, \\
-g_{22}=r^{2} B & =r^{2}\left(1+\sum_{p=2}^{\infty} \sum_{s=2}^{\infty} e^{p} a^{s} \stackrel{(p s)}{B}\right), \\
-g_{33}=r^{2} \sin ^{2} \theta C & =r^{2} \sin ^{2} \theta\left(1+\sum_{p=2}^{\infty} \sum_{s=2}^{\infty} e^{p} a^{s} C^{(p s)}\right), \\
g_{44}=D & =1+\sum_{p=2}^{\infty} \sum_{s=2}^{\infty} e^{p} a^{s} D,
\end{aligned}
$$

$\stackrel{(p s)}{A} \stackrel{(p s)}{B}, \stackrel{(p s)}{C}, \stackrel{(p s)}{D}$ being functions of $r, \theta, t$. 


\section{Solution of the Potential Wave Equation}

For the derivation of a formula for the energy tensor $E_{i k}-$ and, in particular, for $\stackrel{(22)}{i k}_{i k}$ required in the setting up of the (22) approximation it is necessary to calculate the electromagnetic 4-potential $\phi_{i}$.

Consider first any isolated electrically charged distribution. It is shown in EDdington $(1924, \S 74)$ that $\phi_{i}$ satisfies the equation ${ }^{1}$

$$
g^{a b} \phi_{i ; a b}=4 \pi\left(J_{i}-R_{i}^{k} \phi_{k}\right) \text {, }
$$

$J_{i}$ being the 4 -current, if we impose the condition

$$
\phi_{; i}^{i}=0 \text {. }
$$

For weak fields, $\phi_{k}, R_{i}^{k}$ are small, so that, to the first approximation, equation (5.1) reduces to

$$
g^{a b} \phi_{i ; a b}=4 \pi J_{i}
$$

In Galilean coordinates equations (5.3) and (5.2) are the familiar wave and gauge equations

$$
\begin{aligned}
e_{k} \phi_{i, k k} & =4 \pi J_{i}, \\
e_{i} \phi_{i, i} & =0,
\end{aligned}
$$

where $e_{i}=-1,-1,-1,+1$, terms of small magnitude of order higher than the first being neglected; equations (5.4) and (5.5) are thus the linearized forms of equations (5.1) and (5.2) in Galilean coordinates.

The Kirchhoff solution of equations (5.4) and (5.5), for the general case in which the sources of the field simultaneously emit and absorb radiation, is

where

$$
\phi_{i}=\alpha \stackrel{(-)}{\phi}_{i}+\beta \stackrel{(+)}{\phi}_{i}(\alpha+\beta=1)
$$

$$
\stackrel{(\mp)}{\phi_{i}}=\int_{V} r^{*-1} J_{i}\left(\tilde{x}, \tilde{y}, \tilde{z}, t \mp r^{*}\right) d v, \quad e_{i} J_{i, i}=0 .
$$

The notation in this form of solution is as follows: In equation (5.6), $\stackrel{(-)}{\phi}_{i}$ and $\stackrel{(+)}{\phi}_{i}$ represent respectively the retarded-potential and advancedpotential solutions of equations (5.4) and (5.5) and correspond to the radiation emitted from, and absorbed by, the sources of the field; $\alpha, \beta$ are non-negative constants, which will be referred to as the strengths of the emitted and absorbed radiation, respectively. In the first of equations (5.7) the integration is taken over any fixed space volume $V$ containing all the sources of the field and $r^{*}$ is the distance of the point $\widetilde{P}(\tilde{x}, \tilde{y}, \tilde{z})$,

1 In this paper a Latin index runs from 1 to 4 , a Greek one from 1 to 3 ; the summation convention applies to both types of index. A semicolon subscript denotes covariant differentiation, a comma subscript indicates partial differentiation. 
contained by the space element $d v=d \tilde{x} d \tilde{y} d \tilde{z}$ of integration, from the field-point $P(x, y, z)$ of interest; the second of equations (5.7), deducible from equations (5.4) and (5.5), simply expresses the law of conservation of $J_{i}$.

Before applying the solution (5.6) and (5.7) to the dipole of section 2 it will be convenient to re-express the solution so that $r$ occurs in place of $r^{*}, r$ being the fixed distance $O P$. On applying the result to the source and introducing the notation (4.2) we obtain the following (multipole) expansion for the solution of equations (5.4) and (5.5) in terms of $e a^{s}$ $(s=1,2,3, \ldots)$ (see appendix I); only the leading term of each nonvanishing $\phi_{i}$ is written out explicitly:

$$
\left.\begin{array}{l}
\phi_{1}=\phi_{2}=0 \\
\phi_{3}=-2 e a r^{-1} \bar{f}^{\prime}+O\left(e a^{2}\right), \\
\phi_{4}=2 e a \cos \theta\left(r^{-1} \hat{f}^{\prime}+r^{-2} \bar{f}\right)+O\left(e a^{2}\right)
\end{array}\right\}
$$

valid for $r>\max .|\zeta|$, where the notation (III.1) of appendix III has been used for $f(t)$.

\section{The Electromagnetic Energy Tensor and the Flux of Energy}

For the dipole the components of the 4-potential in spherical polar coordinates are, on account of equations (5.8),

$$
\left.\begin{array}{l}
\phi_{1}=-2 e a r^{-1} \cos \theta \bar{f}^{\prime}+O\left(e a^{2}\right), \\
\phi_{2}=2 e a \sin \theta \bar{f}^{\prime}+O\left(e a^{2}\right), \\
\phi_{3}=0 \\
\phi_{4}=2 e a \cos \theta\left(r^{-1} \hat{f}^{\prime}+r^{-2} \bar{f}\right)+O\left(e a^{2}\right)
\end{array}\right\} .
$$

The components of the electromagnetic force tensor for any source are given by the formula

$$
F_{i k}=\phi_{i, k}-\phi_{k, i} \text {; }
$$

for the dipole, the values of the non-zero covariant components in spherical polar coordinates turn out to be

$$
\left.\begin{array}{l}
F_{12}=-F_{21}=2 e a \sin \theta\left(\hat{f}^{\prime \prime}+r^{-1} \bar{f}^{\prime}\right)+O\left(e a^{2}\right), \\
F_{14}=-F_{41}=4 e a \cos \theta\left(r^{-2} \hat{f}^{\prime}+r^{-3} \bar{f}\right)+O\left(e a^{2}\right), \\
F_{24}=-F_{42}=2 e a \sin \theta\left(\bar{f}^{\prime \prime}+r^{-1} \hat{f}^{\prime}+r^{-2} \bar{f}\right)+O\left(e a^{2}\right),
\end{array}\right\}
$$

and the non-vanishing contravariant components are given in terms of the covariant ones as ${ }^{2}$

$$
\left.\begin{array}{l}
F^{12}=-F^{21}=r^{-2} F_{12}+O\left(e a^{2}\right), \\
F^{14}=-F^{41}=-F_{14}+O\left(e a^{2}\right), \\
F^{24}=-F^{42}=-r^{-2} F_{24}+O\left(e a^{2}\right)
\end{array}\right\} .
$$

${ }^{2}$ Henceforth, the notation $O\left(e^{p} a^{s}\right)$ indicates a double power series in $e$ and $a$ consisting of terms of orders $e^{p} a^{r}(r \geqq s)$ and $e^{q} a^{r}(q>p, r \geqq 0)$. 
The formula for any source for the components of the electromagnetic energy tensor is

$$
E_{l k}^{i}=-F^{i a} F_{k a}+\frac{1}{4} \delta_{k}^{i} F^{a b} F_{a b} .
$$

From equations (6.5), (6.3) and (6.4) we find for the non-vanishing $E_{i k}$ in spherical polar coordinates the following expressions for our special source:

$$
\begin{aligned}
E_{11}= & 2 e^{2} a^{2}\left[r^{-2} s^{2}\left(\bar{f}^{\prime \prime 2}+\bar{f}^{\prime \prime 2}\right)+2 r^{-3} s^{2}\left(\bar{f}^{\prime} \hat{f}^{\prime \prime}+\hat{f}^{\prime} \bar{f}^{\prime \prime}\right)+\right. \\
& +r^{-4}\left\{s^{2}\left(\bar{f}^{\prime 2}+\hat{f}^{\prime 2}+2 \bar{f} \bar{f}^{\prime \prime}\right)-4 c^{2} \hat{f}^{\prime 2}\right\}- \\
& \left.-\left(4-5 s^{2}\right)\left(2 r^{-5} \bar{f} \hat{f}^{\prime}+r^{-6} \bar{f}^{2}\right)\right]+O\left(e^{2} a^{3}\right) \\
r^{-2} E_{22}= & 2 e^{2} a^{2}\left[-r^{-2} s^{2}\left(\bar{f}^{\prime \prime 2}-\hat{f}^{\prime \prime 2}\right)+2 r^{-3} s^{2}\left(\bar{f}^{\prime} \hat{f}^{\prime \prime}-\hat{f}^{\prime} \bar{f}^{\prime \prime}\right)+\right. \\
& +r^{-4}\left\{s^{2}\left(\bar{f}^{\prime 2}-\hat{f}^{\prime 2}-2 \bar{f} \bar{f}^{\prime \prime}\right)+4 c^{2} \hat{f}^{\prime 2}\right\}+ \\
& \left.+\left(4-5 s^{2}\right)\left(2 r^{-5} \bar{f}^{\prime}+r^{-6} \bar{f}^{2}\right)\right]+O\left(e^{2} a^{3}\right) \\
r^{-2} s^{-2} E_{33}= & 2 e^{2} a^{2}\left[r^{-2} s^{2}\left(\bar{f}^{\prime \prime 2}-\hat{f}^{\prime \prime 2}\right)-2 r^{-3} s^{2}\left(\bar{f}^{\prime} \hat{f}^{\prime \prime}-\hat{f}^{\prime} \bar{f}^{\prime \prime}\right)+\right. \\
& +r^{-4}\left\{-s^{2}\left(\bar{f}^{\prime 2}-\hat{f}^{\prime 2}-2 \bar{f}^{\prime \prime}\right)+4 c^{2} \hat{f}^{\prime 2}\right\}+ \\
& \left.+\left(4-3 s^{2}\right)\left(2 r^{-5} \bar{f}^{\prime}+r^{-6} \bar{f}^{2}\right)\right]+O\left(e^{2} a^{3}\right) \\
E_{44}= & 2 e^{2} a^{2}\left[r^{-2} s^{2}\left(\bar{f}^{\prime \prime 2}+\hat{f}^{\prime \prime 2}\right)+2 r^{-3} s^{2}\left(\bar{f}^{\prime} \hat{f}^{\prime \prime}+\hat{f}^{\prime} \bar{f}^{\prime \prime}\right)+\right. \\
& +r^{-4}\left\{s^{2}\left(\bar{f}^{\prime 2}+\hat{f}^{\prime 2}+2 \bar{f} \bar{f}^{\prime \prime}\right)+4 c^{2} \hat{f}^{\prime 2}\right\}+ \\
& \left.+\left(4-3 s^{2}\right)\left(2 r^{-5} \bar{f}^{\prime}+r^{-6} \bar{f}^{2}\right)\right]+O\left(e^{2} a^{3}\right) \\
r^{-1} E_{12}= & -8 e^{2} a^{2} s c\left[r^{-3} \hat{f}^{\prime} \bar{f}^{\prime \prime}+r^{-4}\left(\bar{f} \bar{f}^{\prime \prime}+\hat{f}^{\prime 2}\right)+\right. \\
& \left.+\left(2 r^{-5} \bar{f} \hat{f}^{\prime}+r^{-6} \bar{f}^{2}\right)\right]+O\left(e^{2} a^{3}\right), \\
E_{14}= & -4 e^{2} a^{2} s^{2}\left[r^{-2} \bar{f}^{\prime \prime} \hat{f}^{\prime \prime}+r^{-3}\left(\bar{f}^{\prime} \bar{f}^{\prime \prime}+\hat{f}^{\prime} \hat{f}^{\prime \prime}\right)+\right. \\
& \left.+r^{-4}\left(\bar{f} \hat{f}^{\prime \prime}+\bar{f}^{\prime} \hat{f}^{\prime}\right)+r^{-5} \bar{f}^{\prime}\right]+O\left(e^{2} a^{3}\right) \\
r^{-1} E_{24}= & 8 e^{2} a^{2} s c\left[r^{-3} \hat{f}^{\prime} \hat{f}^{\prime \prime}+r^{-4}\left(\bar{f} \hat{f}^{\prime \prime}+\bar{f}^{\prime} \hat{f}^{\prime}\right)+\right. \\
& \left.+r^{-5} \bar{f} \bar{f}^{\prime}\right]+O\left(e^{2} a^{3}\right)
\end{aligned}
$$

where $s=\sin \theta, c=\cos \theta$.

We now calculate the total flux of energy of electromagnetic radiation from the dipole across a sphere $S$ with centre $O$ and radius $R$ : on account of equations (6.11) and (4.3) it is given by

$$
\begin{aligned}
& F_{E(\infty)}=\int_{-\infty}^{\infty} d t \int_{S} E^{14} d S \\
& =\frac{32 \pi}{3}(\alpha-\beta) e^{2} a^{2} \int_{t_{1}}^{t_{2}} f^{\prime \prime 2}(\xi) d \xi+O\left(R^{-1}\right)+O\left(e^{2} a^{3}\right) \quad(\alpha+\beta=1) .(6
\end{aligned}
$$

The total energy flowing out of an infinite sphere, centre $O$, is therefore

$$
\frac{32 \pi}{3}(\alpha-\beta) e^{2} a^{2} \int_{t_{1}}^{t_{2}} f^{\prime \prime 2}(\xi) d \xi+O\left(e^{2} a^{3}\right) \quad(\alpha+\beta=1) .
$$


This, for the general case $\alpha \neq \beta$, does not vanish since $f^{\prime \prime}(\xi) \neq 0$, uniform motion of the charges having been ruled out. Hence, provided the waves are not stationary one expects a permanent variation in mass of the oscillating dipole, of an amount minus the value (6.14), to show up in the (22) approximation to the metric ${ }^{3}$. This is confirmed in the next section.

\section{The Solution of the (22) Approximation. Change of Mass of the Source}

The (22) approximation is equation (4.4) with $p=s=2$ and with $\stackrel{(22)}{\Psi}_{l m}=0$; explicitly, for the metric (3.1) it becomes equations (II.1) to (II.7) of appendix II in which the quantities $\stackrel{(22)}{P}, \stackrel{(22)}{Q}, \ldots, \stackrel{(22)}{N}$ on the right are given by

$$
\begin{aligned}
& \stackrel{(22)}{P}=-16 \pi \stackrel{(22)}{E}_{11}, \\
& \stackrel{(22)}{Q}=-16 \pi r^{-2} \stackrel{(22)}{E}_{22}, \\
& \stackrel{(22)}{R}=-16 \pi r^{-2} s^{-2} \stackrel{(22)}{E}_{33}, \\
& \stackrel{(22)}{S}=-16 \pi \stackrel{(22)}{H}_{44},
\end{aligned}
$$

and equations (6.6) to (6.12).

The complete solution of the $(p s)$ approximation is given by equations (II.8) to (II.11). It contains six functions of integration $\eta^{(p s)}(r, \theta)$, $\stackrel{(p s)}{\sigma}(r, 0), \stackrel{(p s)}{\chi}(r, t), \stackrel{(p s)}{\nu}(\theta, t), \stackrel{(p s)}{\tau}(\theta, t), \stackrel{(p s)}{\mu}(r, t)$, only one of which, $\stackrel{(p s)}{\chi}(r, t)$, to be given a non-zero value in order to satisfy the two requirements laid down in the paragraph containing equation (II.13) of appendix II. The key to this solution is the value of $\stackrel{(p s)}{A}$ satisfying the inhomogeneous wave equation (II.8), which is inserted here for convenient reference:

$$
\begin{aligned}
& \square \stackrel{(p s) \mathrm{def}}{=}\left(\stackrel{(p s)}{A_{11}}+2 r^{-1} \stackrel{(p s)}{A}_{1}\right)+r^{-2}\left(\stackrel{(p s)}{A_{22}}+\stackrel{(p s)}{A_{2}} \cot \theta\right)-\stackrel{(p s)}{A_{44}} \\
& =\stackrel{(p s)}{P}-\int\left(\stackrel{(p s)}{M}_{1}+r^{-1} \stackrel{(p s)}{M}\right) d t-\int\left[\left(\stackrel{(p s)}{L}_{1}+r^{-1} \stackrel{(p s)}{L}\right)-\int\left(\stackrel{(p s)}{N}_{11}+r^{-1} \stackrel{(p s)}{N}_{1}\right) d t\right] d \theta- \\
& -\left(\stackrel{(p s)}{\eta_{1}}+r^{-1} \stackrel{(p s)}{\eta}\right)+\int\left({\stackrel{(p s)}{\sigma_{11}}}^{\prime}+r^{-1} \sigma_{1}^{(p s)}\right) d \theta-\left({ }^{(p s)}+r_{1}^{-1} \stackrel{(p s)}{\chi}\right) \text {. }
\end{aligned}
$$

Obtaining an appropriate exact solution of the (22) approximation is extremely difficult: to avoid prohibitive calculations we shall derive a suitable approximate one satisfying the (22) approximation up to terms of order $r^{-3}$, i.e. a solution such that its insertion back into the (22) field

${ }^{3}$ From the expansion (4.3) it is clear that in Galilean coordinates the components $\mathrm{t}_{k}^{i}$ of the energy pseudo-tensor are of order $e^{4} a^{4}$. This should be sufficient to indicate that the energy of gravitational radiation accompanying electromagnetic radiation from the dipole is of order $e^{4} a^{4}$ and so may be overlooked, as the components $E_{k}^{i}$ of the electromagnetic energy tensor for the dipole are of order $e^{2} a^{2}$ only. 
equations leads to the cancellation of terms up to order $r^{-3}$. The reason for this step is as follows:

Suppose that the occurrence of electromagnetic waves is accompanied by a secular variation in the mass of the source. Then the form of the Schwarzschild solution shows that for large $r$ the coefficient of $r^{-1}$ in the metric for the dipole after the end of its oscillation should differ from that before the beginning. It is therefore the $r^{-1}$ terms in the solution of the (22) approximation that we are interested in, and they do not produce terms of order exceeding $r^{-3}$ on the left of equations (II.1) to (II.7) $(p=s=2)$. Hence we assume that it is sufficient to obtain a suitable approximate solution of the (22) approximation (II.1) to (II.7) $(p=s=2$ ) satisfying the latter up to terms of order $r^{-3}$. In any case this will ensure that up to the (22) approximation the total flux of 'phoney' matter across a large sphere with centre $O$ and radius $R$ (got by using the material energy tensor $T_{i k}$ ) is of order $R^{-2}$, i.e. zero across an infinite sphere. Thus the approximate solution and the corresponding exact solution of the (22) approximation should give the same numerical result as far as the total variation in mass of the source is concerned (cf. BonNor, 1959, §11 for the case of gravitational waves).

In accordance with the above we need values for the quantities (7.1) only up to terms in $r^{-3}$ : from the formulae (6.6) to (6.12) they are

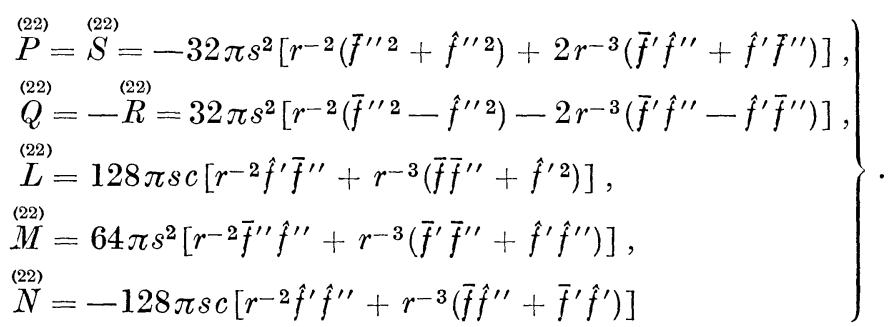

It will save a great amount of work if the expressions (7.3) are each broken up into two parts, as below:

where

$$
\stackrel{(22)}{P}=\stackrel{(22)}{P}+\underset{1}{\stackrel{(22)}{P}}, \quad \text { etc. }
$$

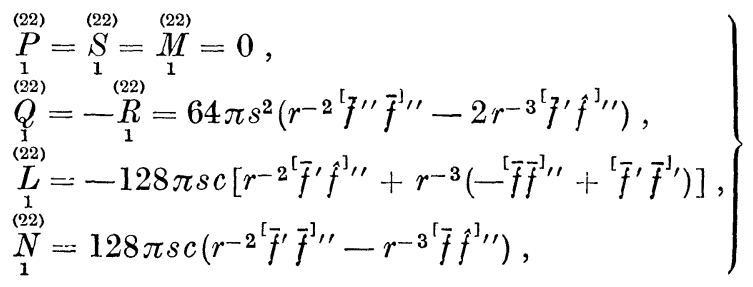




$$
\left.\begin{array}{l}
\stackrel{(22)}{P}=\underset{2}{S}=-64 \pi s^{2}\left(r ^ { - 2 } \left(\bar{f}^{\prime \prime} \bar{f}^{\prime \prime}+2 r^{-3}\left(\bar{f}^{\prime} \hat{f}^{\prime \prime}\right),\right.\right. \\
\underset{2}{(22)}=\underset{2}{(22)}=0 \\
\underset{2}{R}=\underset{2}{(22)}=0 \\
\underset{2}{L}=128 \pi s c\left[r ^ { - 2 } \left(\bar{f}^{\prime} \hat{f}^{\prime \prime \prime}+r^{-3}\left(\bar{f} \bar{f}^{\prime \prime \prime}+\left(\bar{f}^{\prime} \bar{f}^{\prime}\right)\right],\right.\right. \\
\stackrel{(22)}{M}=64 \pi s^{2}\left(r ^ { - 2 } \left(\bar{f}^{\prime \prime} \hat{f}^{\prime \prime}+2 r^{-3}\left(\bar{f}^{\prime} \bar{f}^{\prime \prime \prime}\right),\right.\right. \\
\underset{2}{(22)}=-128 \pi s c\left[r ^ { - 2 } \left(\bar{f}^{\prime} \bar{f}^{\prime \prime \prime}+r^{-3}\left(\bar{f} \hat{f}^{\prime \prime \prime}+\left(\bar{f}^{\prime} \hat{f}^{\prime}\right)\right]\right.\right.
\end{array}\right\} .
$$

Here, the notations (III.2) and (III.3), as applied to $f$, are assumed. The above treatment for $\stackrel{(22)}{P}, \stackrel{(22)}{Q}, \ldots, \stackrel{(22)}{N}$ automatically leads to a similar splitting of equations (II.1) to (II.7) $(p=s=2)$ (which are linear in the $\stackrel{(22)}{g}_{i k}$ and their derivatives) into two sets of equations (I) and (II), having for the right-hand sides the expressions (7.5) and (7.6), respectively.

Our object now is to obtain suitable approximate solutions of equations (I) and (II) satisfying them up to order $r^{-3}$. To avoid possible confusion we shall distinguish the corresponding $\stackrel{(22)}{g}_{i k}$, occurring on the left of equations (I) and (II), by designating them $\underset{1}{\operatorname{as}} \underset{g_{i k}}{(22)}$ and $\underset{2}{\stackrel{(22)}{g}}$, respectively.

The key equation (7.2) ( $p=s=2$ ) will now consist of two parts: by making use of the formulae (7.5) and (7.6) these become, up to order $r^{-3}$,

$$
\begin{aligned}
& \square \underset{(22)}{\stackrel{(22)}{A}=128 \pi r^{-3} s^{2}\left[T^{\prime} \hat{f}^{]}, \prime,\right.} \\
& \square \stackrel{(22)}{A}=64 \pi r^{-3} s^{2}\left(-2\left(\bar{f}^{\prime} \hat{f}^{\prime \prime \prime}+\hat{\hat{Y}}\right)-\left(\stackrel{(22)}{\chi_{1}}+r^{-1}{ }^{(22)} \chi\right)\right. \text {; }
\end{aligned}
$$

in equation (7.8) the notation (III.7) has been used to introduce the symbol $\hat{\hat{Y}}$, where $Y(\xi)$ is defined by

$$
Y^{\prime}(\xi)=X(\xi)=f^{\prime \prime 2}(\xi) \text {. }
$$

In obtaining equation (7.7) we put all the arbitrary functions $\stackrel{(22)}{\eta}, \stackrel{(22)}{\sigma}, \stackrel{(22)}{\chi}$ equal to zero, whereas in obtaining equation (7.8) we retained ${ }^{(22)} \chi$ for use later to preclude singularities from appearing along $O z$ (except at $O$ ).

For a solution of equation (7.7) it is found necessary to proceed as far as the term of order $r^{-3}$ in the calculation of $\underset{1}{A}$, if the corresponding complete solution of equations (I) is to satisfy the latter up to order $r^{-3} .^{4}$ For equation (7.8), however, the situation is somewhat different. It turns out that, to obtain a solution of equations (II) satisfying them up to order $r^{-3}$, it is sufficient to solve equation (7.8) for $\underset{2}{A}$ only to order $r^{-1}$. In the expressions for $\underset{2}{B}, \underset{2}{C}, \underset{2}{D}$, found with the aid of equations (II.9)

4 This solution of equations (I) will itself contain only terms of order $r^{-2}$ or higher, as we shall soon find (see the solution (7.11)), and will accordingly be neglected in due course. 
to (II.11), terms of order $r^{-2}$ and $r^{-3}$ must, nevertheless, be retained, except those arising from similar terms in $\underset{2}{A}$, which will have been ignored in any case. Combining the two solutions of equations (I) and (II) obtained to the degree of approximation required above, one will have a solution satisfying the (22) approximation up to order $r^{-3}$.

It is easily verified that

$$
\stackrel{(22)}{A}=-32 \pi r^{-3} s^{2}\left[\bar{f} \hat{f}^{]}\right.
$$

satisfies equation (7.7) up to order $r^{-3}$, and from this and equations (II.9) to (II.11) the remaining non-zero $\stackrel{(22)}{g}_{i k}$ can readily be obtained. The corresponding complete solution of equations $(\mathrm{I})$ is as follows (after some partial integration):

$$
\begin{aligned}
& \frac{1}{32 \pi} \stackrel{(22)}{A}=-r^{-3} s^{2}\left[-\hat{f}^{]},\right. \\
& \frac{1}{32 \pi} \stackrel{(22)}{B}=s^{2}\left(-\frac{1}{2} r^{-2}\left[\bar{f}^{\prime} \bar{f}^{-}\right]^{\prime}+\frac{1}{2} r^{-3}\left[\bar{f} \hat{f}^{]}\right)\right. \text {, } \\
& \frac{1}{32 \pi} \stackrel{(22)}{C}=s^{2}\left(\frac { 1 } { 2 } r ^ { - 2 } \left[\bar{f}^{\prime} \bar{f}^{]}-\frac{1}{2} r^{-3}\left[\bar{f} \hat{f}^{] \prime}\right),\right.\right. \\
& \frac{1}{32 \pi} \stackrel{(22)}{D}=s^{2}\left(-r^{-3}\left[-\left[-\hat{f}^{\prime}{ }^{\prime}+2 r \int_{\infty}^{r} r^{-4}\left[\bar{f}^{\prime} \bar{f}^{-} d r\right)\right.\right.\right.
\end{aligned}
$$

For verifying that this solution satisfies equations (I) up to order $r^{-3}$ it is necessary to differentiate the integral in the expression for $\underset{1}{D}$, with respect to $t$, through the sign of integration. This is in order, since this integral is uniformly convergent for all $t$, if $r>0$, as is readily seen by the Weierstrass $M$-test for integrals.

All the terms in the above solution of equations (I) are of order $r^{-n}(n \geqq 2)$ and will therefore represent no permanent change of order $r^{-1}$ in the metric. Hence, our main concern is to obtain a solution of equations (II) to the required degree of approximation, starting with the wave equation (7.8).

A solution of equation (7.8) can be built up from solutions of equations of the form

$$
\square \Phi=r^{-3} s(\theta)\left(\bar{f}^{\prime} \hat{f}^{\prime \prime}, \quad \square \Phi=r^{-3} s(\theta) \hat{\hat{Y}} .\right.
$$

It is easily verifiable that the functions

$$
\Phi=r^{-1} \stackrel{1}{S}(\theta) \text { ' } \tilde{f}^{\prime} \hat{f}^{\prime \prime}, \quad \Phi=r^{-1} \stackrel{2}{S}(\theta) \hat{Y}
$$

are respectively (exact) solutions of equations (7.12) if

$$
\stackrel{\alpha}{S_{22}}+\stackrel{\alpha}{S_{2}} \cot \theta=\stackrel{\alpha}{s} \quad(\alpha=1,2)
$$

Unfortunately, it is not in general possible to obtain solutions of equations (7.14) which are non-singular for $0 \leqq \theta \leqq \pi$. This difficulty can be overcome, however, by means of the arbitrary function ${ }^{(22)}(r, t)$, specially 
retained in equation (7.8) for this purpose. Consider then the equations

$$
\left.\begin{array}{l}
\square \Phi=r^{-3} s(\theta)\left(\bar{f}^{\prime} \hat{f}^{\prime \prime \prime}-\left(\begin{array}{c}
(22) \\
\chi \\
1
\end{array}+r^{-1}{ }^{(22)} \chi\right),\right. \\
\square \Phi=r^{-3} s(\theta) \hat{Y}-\left(\begin{array}{c}
(22) \\
\chi_{1}+r^{-1} \chi
\end{array}\right)
\end{array}\right\}
$$

and choose $\stackrel{(22)}{\chi}(r, t)$ such that

$$
\stackrel{(22)}{\chi_{1}}+r^{-1} \underset{\chi}{\chi}=k_{1} r^{-3}\left(\bar{f}^{\prime} \hat{f}^{\prime \prime \prime}, k_{2} r^{-3} \hat{Y}\right.
$$

in the first and second wave equation, respectively. Employing again the functions (7.13) in equations (7.15) gives

$$
\stackrel{\alpha}{S_{22}}+\stackrel{\alpha}{S} \cot \theta=\stackrel{\alpha}{s}-k_{\alpha} \quad(\alpha=1,2)
$$

instead of equations (7.14), and suitable values for the constants $k_{1}$ and $k_{2}$ can be found so that equations (7.17) possess non-singular solutions for $0 \leqq \theta \leqq \pi$.

By this method we can obtain a solution of equation (7.8) (see below), non-singular in $\theta$, with $\stackrel{(22)}{\chi}(r, t)$ satisfying

$$
\frac{1}{32 \pi}\left(\stackrel{(22)}{\chi}_{1}+r^{-1} \stackrel{(22)}{\chi}\right)=r^{-3}\left(-\frac{8}{3}\left(\bar{f}^{\prime} \hat{f}^{\prime \prime}+\frac{4}{3} \hat{\hat{Y}}\right)\right. \text {. }
$$

For the remaining non-zero $\stackrel{(22)}{g}_{2}$ the values of $\underset{2}{B}, \underset{2}{\stackrel{(22)}{C}} \underset{2}{D}$ can now be calculated up to order $r^{-3}$ by means of equations (II.9) to (II.11). It must be emphasized at this stage that the function $\underset{(22)}{\chi}$, satisfying equation (7.18), occurs again in the formula (II.11) for $\underset{2}{D}$. With the remaining functions of integration put equal to zero, a complete solution of equations (II) is found in the above manner to be (after several partial integrations are carried out)

$$
\begin{aligned}
& \frac{1}{32 \pi} \underset{2}{A}=r^{-1} s^{2}\left(\frac{2}{3}\left(\bar{f}^{\prime} \hat{f}^{\prime \prime \prime}-\frac{1}{3} \hat{\hat{Y}}\right)\right. \text {, } \\
& \frac{1}{32 \pi} \underset{2}{\stackrel{(22)}{B}}=s^{2}\left(\frac { 1 } { 3 } r ^ { - 1 } \left(\bar{f}^{\prime} \hat{f}^{\prime \prime}-\frac{1}{6} r^{-1} \hat{Y}+\right.\right. \\
& +r^{-3}\left(-\bar{f} \hat{f}^{\prime}+r^{-1} \int_{\infty}^{r} r^{-1} \hat{Y} d r+r^{-1} \int_{\infty}^{r} r^{-2}\left(\bar{f}^{\prime} \vec{f}^{\prime} d r\right),\right. \\
& \frac{1}{32 \pi} \stackrel{(22)}{C}_{2}=s^{2}\left(-\frac{1}{3} r^{-1}\left(\bar{f}^{\prime} \hat{f}^{\prime \prime \prime}+\frac{1}{6} r^{-1} \hat{Y}-\frac{2}{3} r^{-2}\left(\bar{f}^{\prime} \bar{f}^{\prime}-r^{-3}\left(\bar{f} \hat{f}^{\prime}+\right.\right.\right.\right. \\
& +\frac{1}{3} r^{-1} \int_{\infty}^{r} r^{-1} \hat{\hat{Y}} d r+\frac{1}{3} r^{-1} \int_{\infty}^{r} r^{-2}\left(-\bar{f}^{\prime} \bar{f}^{\prime} d r\right) \text {, } \\
& \frac{1}{32 \pi} \stackrel{(22)}{D}=\frac{2}{3} r^{-1} s^{2}\left(\bar{f}^{\prime} \hat{f}^{\prime \prime \prime}+\frac{2}{3} r^{-1} \hat{Y}-\frac{2}{3} r^{-2} s^{2} \bar{f}^{\prime}-\bar{f}^{\prime}+\right. \\
& +\frac{4}{3} \int_{\infty}^{r} r^{-1} \overline{\bar{X}} d r-r\left(\frac{2}{3}-\frac{1}{3} s^{2}\right) \int_{\infty}^{r} r^{-2} \overline{\bar{X}} d r- \\
& -\frac{8}{3} \int_{\infty}^{r} r^{-3}\left(\bar{f}^{\prime} \bar{f}^{\prime} d r+4 r c^{2} \int_{\infty}^{r} r^{-4}\left(\bar{f}^{\prime}-\bar{f}^{\prime} d r\right.\right.
\end{aligned}
$$

3 Commun. math. Phys., Vol. 5 
where the notation (III.5) has been employed for the introduction of $\bar{X}, X(\xi)$ being defined by the second equality of (7.9). In the verification that this solution satisfies equations (II) up to order $r^{-3}$ it is necessary to differentiate various integrals with respect to $t$ through the sign of integration. This is allowed on account of the fact that those integrals are uniformly convergent for finite $t$ when $r>0$, as can be shown immediately by the Weierstrass $M$-test.

Combining the two solutions (7.11) and (7.19) of equations (I) and (II), respectively, we obtain, as a suitable solution of the (22) approximation satisfying the latter up to terms of order $r^{-3}$,

$$
\begin{aligned}
& \frac{1}{32 \pi} \stackrel{(22)}{A}=-\frac{1}{3} r^{-1} s^{2} \hat{Y}+R_{A}, \\
& \frac{1}{32 \pi} \stackrel{(22)}{B}=s^{2}\left(-\frac{1}{6} r^{-1} \hat{Y}+r^{-1} \int_{\infty}^{r} r^{-1} \hat{Y} d r\right)+R_{B}, \\
& \frac{1}{32 \pi} \stackrel{(22)}{C}=s^{2}\left(\frac{1}{6} r^{-1} \hat{Y}+\frac{1}{3} r^{-1} \int_{\infty}^{r} r^{-1} \hat{Y} d r\right)+R_{C}, \\
& \frac{1}{32 \pi} \stackrel{(22)}{D}=\frac{2}{3} r^{-1} \hat{Y}+\frac{4}{3} \int_{\infty}^{r} r^{-1} \bar{X} d r-r\left(\frac{2}{3}-\frac{1}{3} s^{2}\right) \int_{\infty}^{r} r^{-2} \bar{X} d r+R_{D} .
\end{aligned}
$$

Here, $R_{A}, \ldots, R_{D}$ (which, incidentally, together include all the terms of the solution (7.11) of equations (I)) consist entirely of terms each of which is either of order $r^{-2}$ or higher for all $t$, or of order $r^{-1}$ and tends to zero as $t \rightarrow \pm \infty$; so $R_{A}, \ldots$ do not contribute to any permanent change of order $r^{-1}$ in the metric.

This solution (7.20) is difficult to interpret physically. However, the coordinate transformation

$$
\begin{aligned}
& r=r^{*}-32 \pi e^{2} a^{2}\left[\left(\frac{1}{3}-\frac{1}{6} s^{* 2}\right) \int_{\infty}^{r^{*}} \eta^{-1} \hat{Y}\left(\eta, t^{*}\right) d \eta+\right. \\
& \left.\quad+r^{*}\left(\frac{1}{6}-\frac{1}{4} s^{* 2}\right) \int_{\infty}^{r^{*}} \eta^{-2} \hat{Y}\left(\eta, t^{*}\right) d \eta\right], \\
& \left.\begin{array}{rl}
\theta= & \theta^{*}+32 \pi e^{2} a^{2} s^{*} c^{*} \times \\
& \times\left[\frac{1}{3} r^{*-1} \int_{\infty}^{r^{*}} \eta^{-1} \hat{Y}\left(\eta, t^{*}\right) d \eta+\frac{1}{6} \int_{\infty}^{r^{*}} \eta^{-2} \hat{Y}\left(\eta, t^{*}\right) d \eta\right], \\
\phi= & \phi^{*}, \quad \\
t= & t^{*}-\frac{32 \pi}{3} e^{2} a^{2}\left[\overline{\bar{Y}}\left(r^{*}, t^{*}\right)+r^{*} \int_{\infty}^{r^{*}} \eta^{-1} \hat{\hat{X}}\left(\eta, t^{*}\right) d \eta\right],
\end{array}\right\}
\end{aligned}
$$


where $s^{*}=\sin \theta^{*}, c^{*}=\cos \theta^{*}$ and the notations (III.5) and (III.7) apply to $X(\xi)$ and $Y(\xi)$ of $(7.9)$, brings it to one that can be physically interpreted as we shall soon find. The transformed (22) solution is

$$
\begin{aligned}
& -\frac{1}{32 \pi} \stackrel{(22)}{g}_{11}=\frac{1}{32 \pi} \stackrel{(22)}{A} \\
& =-\frac{2}{3} r^{-1} \hat{\hat{Y}}+\left(\frac{1}{3}-\frac{1}{2} s^{2}\right) \int_{\infty}^{r} \eta^{-1} \overline{\bar{X}}(\eta, t) d \eta-R_{A}, \\
& -\frac{1}{32 \pi} r^{-2} \stackrel{(22)}{g}=\frac{1}{32 \pi} \stackrel{(22)}{B}=\frac{1}{6} s^{2} \int_{\infty}^{r} \eta^{-1} \overline{\bar{X}}(\eta, t) d \eta-R_{B}, \\
& -\frac{1}{32 \pi} r^{-2} s^{-2} \stackrel{(22)}{g}=\frac{1}{32 \pi} \stackrel{(22)}{C}=-\frac{1}{6} s^{2} \int_{\infty}^{r} \eta^{-1} \bar{X}(\eta, t) d \eta-R_{C} \\
& \frac{1}{32 \pi} \stackrel{(22)}{g}=\frac{1}{32 \pi} \stackrel{(22)}{D}=\frac{2}{3} r^{-1} \hat{\hat{Y}}+\frac{4}{3} \int_{\infty}^{r} \eta^{-1} \overline{\bar{X}}(\eta, t) d \eta+ \\
& +\frac{1}{3} r s^{2} \int_{\infty}^{r} \eta^{-2} \overline{\bar{X}}(\eta, t) d \eta+R_{D} \\
& \frac{1}{32 \pi} r^{-1} \stackrel{(22)}{g_{12}}=\frac{1}{32 \pi} \stackrel{(22)}{E}=\frac{1}{2} s c \int_{\infty}^{r} \eta^{-1} \overline{\bar{X}}(\eta, t) d \eta \\
& \frac{1}{32 \pi} \stackrel{(22)}{g}_{14}=\frac{1}{32 \pi} \stackrel{(22)}{F}=-\frac{1}{6} s^{2} \int_{\infty}^{r} \eta^{-1} \hat{\hat{X}}(\eta, t) d \eta+ \\
& +r\left(\frac{1}{6}-\frac{1}{4} s^{2}\right) \int_{\infty}^{r} \eta^{-2} \hat{\hat{X}}(\eta, t) d \eta, \\
& \frac{1}{32 \pi} r^{-1} \stackrel{(22)}{g}=\frac{1}{32 \pi} \stackrel{(22)}{G} \\
& =-s c\left[\frac{1}{3} \int_{\infty}^{r} \eta^{-1} \hat{\hat{X}}(\eta, t) d \eta+\frac{1}{6} r \int_{\infty}^{r} \eta^{-2} \hat{\hat{X}}(\eta, t) d \eta\right],
\end{aligned}
$$

the asterisks being omitted; the previous approximations are unaffected. During the transformation it is necessary to differentiate some of the integrals in equations (7.21) with respect to $t$ through the sign of integration. This is permissible, since the Weierstrass $M$-test can be used to show that these integrals are uniformly convergent for finite $t$, if $r>0$. Involving the same method of differentiation is the verification that the solution (7.22) satisfies up to order $r^{-3}$ the (22) approximation for the non-diagonal metric (3.2), i.e. equations (II.14) to (II.20) with the righthand sides given by the formulae (7.3), when appropriate values for $R_{A}, \ldots, R_{D}$ are used in the process. 
In examining the solution (7.22) for physical interpretation we notice integrals of the types

$$
\int_{\infty}^{r} \eta^{-1} X(\eta, t) d \eta, \quad \int_{\infty}^{r} \eta^{-2} X(\eta, t) d \eta
$$

where $X(r, t)$ stands for $\bar{X}$ or $\hat{X}$. It is easily shown that these exist for all $r>0$ and all $t$; that for any given finite value of $t$ these tend to zero as $r \rightarrow \infty$ at least as rapidly as $r^{-2}$ and $r^{-3}$, respectively; that, from the Weierstrass $M$-test, these are uniformly convergent for $-\infty<t<\infty$, for any given positive value of $r$. As $t \rightarrow \pm \infty$, the integrals (7.23) tend to zero (at least as rapidly as $t^{-1}$ and $t^{-2}$, respectively), as can be seen qualitatively by regarding $X(t \mp \eta)$ as a $\delta$-function; a similar argument shows that they tend to zero as $r$ tends to infinity with $|t|$ in such a way that $t \mp r \rightarrow$ a finite limit.

It follows that the metric (7.22) possesses no singularities for $r>0$. Moreover, owing to the fact that, as $r \rightarrow \infty$ and $t \rightarrow \pm \infty$, the derivatives of the expressions (7.23) tend to zero at least as rapidly as the expressions themselves (this becomes obvious on differentiation), it follows that the metric (7.22) tends to flatness as $r \rightarrow \infty$ and $t \rightarrow \pm \infty$.

Ignoring the expressions (7.23) and $R_{A}, \ldots, R_{D}$ in the solution (7.22), which do not give significant changes in the metric, the solution becomes

$\stackrel{(22)}{g_{11}}=\stackrel{(22)}{g}=\frac{64 \pi}{3} r^{-1} \hat{Y}=\frac{64 \pi}{3} r^{-1}\left[\alpha^{2} \int_{-\infty}^{t-r} f^{\prime \prime 2}(\xi) d \xi-\beta^{2} \int_{\infty}^{t+r} f^{\prime \prime 2}(\xi) d \xi\right]$

according to the notation (III.7) and equations (7.9). If $r(>0)$ is given, then

$$
\stackrel{(2}{g}_{11}: \stackrel{(22)}{g}_{44}= \begin{cases}\frac{64 \pi}{3} \beta^{2} r^{-1} \int_{t_{1}}^{t_{2}} f^{\prime \prime 2}(\xi) d \xi, & \text { for } t<t_{1}-r, \\ \frac{64 \pi}{3} \alpha^{2} r^{-1} \int_{t_{1}}^{t_{2}} f^{\prime \prime 2}(\xi) d \xi, & \text { for } t>t_{2}+r .\end{cases}
$$

The formula (7.25) corresponds to an approximate Schwarzschild metric (with terms in $r^{-2}$ ignored) for a single uncharged particle of mass $\tilde{m}$ given by

$$
\tilde{m}= \begin{cases}-\frac{32 \pi}{3} \beta^{2} e^{2} a^{2} \int_{t_{1}}^{t_{2}} f^{\prime \prime 2}(\xi) d \xi, & \text { for } t<t_{1}-r, \\ -\frac{32 \pi}{3} \alpha^{2} e^{2} a^{2} \int_{t_{1}}^{t_{2}} f^{\prime \prime 2}(\xi) d \xi, & \text { for } t>t_{2}+r .\end{cases}
$$


Thus the above solution of the (22) approximation shows that there occurs a secular variation $\Delta m$ in the mass of the source in this approximation, given by

$$
\Delta m=-\frac{32 \pi}{3}(\alpha-\beta) e^{2} a^{2} \int_{t_{1}}^{t_{2}} f^{\prime \prime 2}(\xi) d \xi \quad(\alpha+\beta=1) .
$$

Up to the (22) approximation this variation in mass is precisely equal to minus the total flux of energy of electromagnetic waves from the source, given by the formula (6.14). As expected, this total variation in mass vanishes when the waves are stationary $\left(\alpha=\beta=\frac{1}{2}\right)$.

\section{Appendix I}

\section{The Multipole Wave Solution}

We derive here the solution (5.8), for the source in section 2 , of the wave and gauge equations (5.4) and (5.5). First we obtain the expansion for the Kirchhoff solution (5.6) and (5.7) (relevant to any electrically charged distribution) in which $r^{*}$ is replaced by $r$.

Carrying out the Taylor expansion about $t \mp r$ for the integrand in the first of equations (5.7) we obtain

$$
\frac{1}{r^{*}} J_{i}\left(\tilde{x}, \tilde{y}, \tilde{z}, t \mp r^{*}\right)=\sum_{n=0}^{\infty} \frac{(\mp 1)^{n}}{n !}\left(\frac{g^{n}}{r^{*}}\right) J_{i}^{(n)}(\tilde{x}, \tilde{y}, \tilde{z}, t \mp r)
$$

(signs corresponding), where

$$
g=r^{*}-r
$$

and the symbol ${ }^{(n)}$ means $\partial^{n} / \partial t^{n}$. Employing the binomial theorem in expanding $g^{n} / r^{*}(n=0,1,2, \ldots)$ in ascending powers of $r^{-1}$ (and $\tilde{r}$ ) for the range $r>\tilde{r}=O \widetilde{P}=\left(\tilde{x}^{2}+\tilde{y}^{2}+\tilde{z}^{2}\right)^{1 / 2}$, we get

$$
\frac{1}{r^{*}}=\frac{1}{r} \sum_{n=0}^{\infty} \frac{\tilde{r}^{n}}{r^{n}} P_{n}\left(\cos \theta^{*}\right), \frac{g}{r^{*}}=-\sum_{n=1}^{\infty} \frac{\tilde{r}^{n}}{r^{n}} P_{n}\left(\cos \theta^{*}\right), \ldots,
$$

in which $\theta^{*}=\Varangle P O \widetilde{P}$ and $P_{n}$ are the Legendre polynomials. Substituting the expansions (I.3) into the expansion (I.1), using the formulae

$$
\tilde{r}^{2}=\tilde{x}_{\lambda} \tilde{x}_{\lambda}, \quad \cos \theta^{*}=x_{\lambda} \tilde{x}_{\lambda} / r \tilde{r},
$$

where $x_{\lambda}=(x, y, z), \tilde{x}_{\lambda}=(\tilde{x}, \tilde{y}, \tilde{z})$, and inserting the result in the first of equations (5.7) we arrive at the following required (multipole) expansion for the right-hand side of equation (5.6):

$$
\phi_{i}=r^{-1} \bar{I}_{i}+x_{\lambda}\left(r^{-2} \hat{I}_{i / \lambda}^{\prime}+r^{-3} \bar{I}_{i / \lambda}\right)+
$$

+ terms involving moments of $J_{i}$ of order higher than the first.

Here, $I_{i / \lambda \mu \nu} \ldots$ are the moments of $J_{i}$ at time $t$ about the coordinate planes, defined as

$$
I_{i / \lambda \mu \nu \ldots}(t)=\int_{V} \tilde{x}_{\lambda} \tilde{x}_{\mu} \tilde{x}_{\nu} \ldots J_{i}(\tilde{x}, \tilde{y}, \tilde{z}, t) d v \quad(\lambda, \mu, v, \ldots=1,2,3),
$$


and the notation (III.1) of appendix III has been used. In this solution the expansion is valid for any point on or outside the smallest sphere, centre $O$, that can enclose for all time all the sources of the field.

For the particular system of section 2 we use the notation (4.2) to obtain readily the solution (5.8), valid for $r>\max .|\zeta|$. This is the required solution for this source, because it satisfies the gauge equation (5.5) as well as the wave equation (5.4) - an expected result since equation (5.5) implies and is implied by the second of equations (5.7), i.e. $e=$ const., for any $\phi_{i}$ satisfying equation (5.4) (MoLLer 1962, § 55).

\section{Appendix II}

\section{The Approximate Field Equations and their Solution}

The $(p s)$ approximation for the diagonal metric (3.1), which is the coefficients of $e^{p} a^{s}$ obtained on insertion of the expansions (4.5) and (4.1) in the field equations (1.1), is written out below. To save printing the labels $(p s)$, which should have been inscribed above the capital letters, have been omitted throughout this appendix, except for those few places where confusion might arise without them.

$$
\begin{aligned}
2 R_{11}^{\prime}=0: & -A_{44}+B_{11}+C_{11}+D_{11}+2 r^{-1}\left(-A_{1}+B_{1}+C_{1}\right)+ \\
& +r^{-2}\left(A_{22}+A_{2} \cot \theta\right)=P \\
2 r^{-2} R_{22}^{\prime}=0: & B_{11}-B_{44}+r^{-1}\left(-A_{1}+3 B_{1}+C_{1}+D_{1}\right)+ \\
& +r^{-2}\left(A_{22}-2 A-B_{2} \cot \theta+2 B+\right. \\
& \left.+C_{22}+2 C_{2} \cot \theta+D_{22}\right)=Q \\
2 r^{-2} s^{-2} R_{33}^{\prime}=0: & C_{11}-C_{44}+r^{-1}\left(-A_{1}+B_{1}+3 C_{1}+D_{1}\right)+ \\
& +r^{-2}\left(A_{2} \cot \theta-2 A-B_{2} \cot \theta+2 B+C_{22}+\right. \\
& \left.+2 C_{2} \cot \theta+D_{2} \cot \theta\right)=R \\
2 R_{44}^{\prime}=0: & A_{44}+B_{44}+C_{44}-D_{11}-2 r^{-1} D_{1}- \\
& -r^{-2}\left(D_{22}+D_{2} \cot \theta\right)=S \\
2 R_{12}^{\prime}=0: & -B_{1} \cot \theta+C_{12}+C_{1} \cot \theta+D_{12}- \\
& -r^{-1}\left(A_{2}+D_{2}\right)=L \\
2 R_{14}^{\prime}=0: & B_{14}+C_{14}+r^{-1}\left(-2 A_{4}+B_{4}+C_{4}\right)=M \\
2 R_{24}^{\prime}=0: & A_{24}-B_{4} \cot \theta+C_{24}+C_{4} \cot \theta=N
\end{aligned}
$$

where $R_{i k}^{\prime}=R_{i k}+8 \pi E_{i k}$. In the above equations a subscript 1,2 or 4 after $A, B, C$ or $D$ indicates differentiation with respect to $r, \theta$ or $t-$ this is to apply to any non-tensorial symbol unless otherwise implied. The left-hand sides of the above equations, corresponding to the $\Phi_{l m}$ in equations (4.4), comprise linear terms in $\stackrel{(p s)}{g}_{i k}$ (and their derivatives); 
$P, Q, \ldots, N$ on the right, corresponding to the right-hand sides of equations (4.4), consist of $E_{i k}$ and the nonlinear expressions $\stackrel{(p s)}{\Psi}_{l m}$ in $\stackrel{(q r)}{g}_{i k}$ (and their derivatives) known from solutions of the lower approximations. For the (22) approximation the $\Psi_{l m}$ vanish and $P, Q, \ldots, N$ are given by the formulae (7.1).

The $(p s)$ approximate field equations (II.1) to (II.7) have already been integrated by BonNor, 1959, in his consideration of gravitational waves, and we simply write down the solution:

$$
\begin{aligned}
\square A \stackrel{\text { def }}{=} & \left(A_{11}+2 r^{-1} A_{1}\right)+r^{-2}\left(A_{22}+A_{2} \cot \theta\right)-A_{44} \\
= & P-\int\left(M_{1}+r^{-1} M\right) d t-\int\left[\left(L_{1}+r^{-1} L\right)-\int\left(N_{11}+r^{-1} N_{1}\right) d t\right] d \theta- \\
& -\left(\eta_{1}+r^{-1} \eta\right)+\int\left(\sigma_{11}+r^{-1} \sigma_{1}\right) d \theta-\left(\chi_{1}+r^{-1} \chi\right), \\
C= & -A+\operatorname{cosec}^{2} \theta \int s c\left[2 A+r^{-1} \int\left\{2 A+r\left(\int M d t+\eta\right)\right\} d r+r^{-1} \tau\right] d \theta+ \\
& +\operatorname{cosec}^{2} \theta \int s^{2}\left(\int N d t+\sigma\right) d \theta+\mu \operatorname{cosec}^{2} \theta, \\
B= & -C+r^{-1} \int\left[2 A+r\left(\int M d t+\eta\right)\right] d r+r^{-1} \tau, \\
D= & A+r \int\left[2 r^{-2} A+r^{-1}\left\{\int\left(L-\int N_{1} d t-\sigma_{1}\right) d \theta+\chi\right\}\right] d r+r v, \text { (II.11) }
\end{aligned}
$$

where

$$
\begin{aligned}
\eta \equiv \eta(r, \theta), \sigma \equiv \sigma(r, \theta), \chi & \equiv \chi(r, t), \\
\nu & \equiv \nu(\theta, t), \tau \equiv \tau(\theta, t), \mu \equiv \mu(r, t)
\end{aligned}
$$

are six functions of integration. The key to this solution of the $(p s)$ approximation is the solution for $A$ of the inhomogeneous wave equation (II.8).

The six functions (II.12) of integration have to be chosen to satisfy two requirements: (i) that the $(p s)$ metric be Galilean at infinity, (ii) that it be non-singular on the rotation axis $O z$, except at $O$. If trivial solutions are ruled out, the necessary and sufficient condition for the satisfaction of the second requirement is that

$$
A, B \operatorname{cosec}^{2} \theta, C \operatorname{cosec}^{2} \theta, D \text { be of class } C^{2} \text { near } \sin \theta=0 .
$$

When using the above solution of the $(p s)$ approximation one must substitute it back into these field equations (II.1) to (II.7) to find out whether further conditions have to be imposed on the six arbitrary functions.

Concerning verification of the solution (7.22) of the (22) approximation we notice that the (22) field equations it satisfies are not in the form (II.1) to (II.7) but correspond to the non-diagonal metric (3.2). The $(p s)$ approximation for this metric is in fact the following equations, 
with $P, Q, \ldots, N$ having similar meanings as before:

$$
\begin{aligned}
& -A_{44}+B_{11}+C_{11}+D_{11}-2 F_{14}+ \\
& +2 r^{-1}\left(-A_{1}+B_{1}+C_{1}+E_{12}+E_{1} \cot \theta\right)+ \\
& +r^{-2}\left(A_{22}+A_{2} \cot \theta+2 E_{2}+2 E \cot \theta\right)=P \text {, } \\
& B_{11}-B_{44}+r^{-1}\left(-A_{1}+3 B_{1}+C_{1}+D_{1}+2 E_{12}-2 F_{4}-2 G_{24}\right)+ \\
& +r^{-2}\left(A_{22}-2 A-B_{2} \cot \theta+2 B+C_{22}+\right. \\
& \left.+2 C_{2} \cot \theta+D_{22}+4 E_{2}+2 E \cot \theta\right)=Q \text {, } \\
& C_{11}-C_{44}+r^{-1}\left(-A_{1}+B_{1}+3 C_{1}+D_{1}+2 E_{1} \cot \theta-2 F_{4}-2 G_{4} \cot \theta\right)+ \\
& +r^{-2}\left(A_{2} \cot \theta-2 A-B_{2} \cot \theta+2 B+C_{22}+2 C_{2} \cot \theta+\right. \\
& \left.+D_{2} \cot \theta+2 E_{2}+4 E \cot \theta\right)=R \text {, } \\
& A_{44}+B_{44}+C_{44}-D_{11}+2 F_{14}+ \\
& +2 r^{-1}\left(-D_{1}+2 F_{4}+G_{24}+G_{4} \cot \theta\right)-r^{-2}\left(D_{22}+D_{2} \cot \theta\right)=S \text {, } \\
& -B_{1} \cot \theta+C_{12}+C_{1} \cot \theta+D_{12}-F_{24}+G_{4}+ \\
& +r\left(E_{44}-G_{14}\right)-r^{-1}\left(A_{2}+D_{2}+2 E\right)=L, \\
& B_{14}+C_{14}+r^{-1}\left(-2 A_{4}+B_{4}+C_{4}+E_{24}+E_{4} \cot \theta+G_{12}+G_{1} \cot \theta\right)+ \\
& +r^{-2}\left(-F_{22}-F_{2} \cot \theta+G_{2}+G \cot \theta\right)=M \text {, } \\
& A_{24}-B_{4} \cot \theta+C_{24}+C_{4} \cot \theta+ \\
& +3 E_{4}+F_{12}-2 G_{1}+r\left(E_{14}-G_{11}\right)=N,
\end{aligned}
$$

in which $\stackrel{\left(p_{s}\right)}{E}, \stackrel{\left(p_{s}\right)}{F}, \underline{(p s)}, \mathrm{G}$, coming from the expansions

$$
\left.\begin{array}{c}
g_{12}=r \sum_{p=2}^{\infty} \sum_{s=2}^{\infty} e^{p} a^{s} \stackrel{(p s)}{E}, \\
g_{14}=\sum_{p=2}^{\infty} \sum_{s=2}^{\infty} e^{p} a^{s} \stackrel{(p s)}{F^{\prime}}, \\
g_{24}=r \sum_{p=2}^{\infty} \sum_{s=2}^{\infty} e^{p} a^{s} \stackrel{(p s)}{G}
\end{array}\right\}
$$

for the non-diagonal coefficients of the metric (3.2), have been included.

\section{Appendix III}

\section{Notation Related to Mixed (Outgoing and Incoming) Radiation}

The notations introduced below for the treatment of combined outgoing and incoming radiation avoid considerable writing in section 7 .

If $\{\psi(\xi)\}$ is a class of functions connected with gravitational or electromagnetic wave fields, then

$$
\left.\begin{array}{l}
\bar{\psi}^{(n)}=\alpha \psi^{(n)}(t-r)+\beta \psi^{(n)}(t+r), \\
\hat{\psi}^{(n)}=\alpha \psi^{(n)}(t-r)-\beta \psi^{(n)}(t+r),
\end{array}\right\}
$$


where ${ }^{(n)}$ denotes $\partial^{n} / \partial t^{n}(n \geqq 0)$.

If $\psi(\xi)$ and $\lambda(\xi)$ are two members of $\{\psi(\xi)\}$, then

$$
\begin{aligned}
& \left.{ }^{[} \bar{\psi}^{(m)} \bar{\lambda}^{3}(p)=-{ }^{[} \hat{\psi}^{(m)} \hat{\lambda}^{\mathrm{I}(p)}=\frac{1}{2}\left(\bar{\psi}^{(m)} \bar{\lambda}^{(p)}-\hat{\psi}^{(m)} \hat{\lambda}^{(m)}\right),\right] \\
& \left.{ }^{[} \bar{\psi}^{(m)} \hat{\lambda}^{]}(p)=-{ }^{[} \hat{\psi}^{(m)} \bar{\lambda}^{-}(p)=\frac{1}{2}\left(\bar{\psi}^{(m)} \hat{\lambda}^{(p)}-\hat{\psi}^{(m)} \bar{\lambda}^{(p)}\right),\right\} \\
& { }^{(} \bar{\psi}^{(m)} \bar{\lambda}^{\prime}(p)=\hat{\psi}^{(m)} \hat{\lambda}^{\prime}(p)=\frac{1}{2}\left(\bar{\psi}^{(m)} \bar{\lambda}^{(p)}+\hat{\psi}^{(m)} \hat{\lambda}^{(p)}\right), \\
& \left.{ }^{\left(\bar{\psi}^{(m)}\right.} \hat{\lambda}^{\prime}(p)={ }^{(} \hat{\psi}^{(m)} \bar{\lambda}^{\prime}(p)=\frac{1}{2}\left(\bar{\psi}^{(m)} \hat{\lambda}^{(p)}+\hat{\psi}^{(m)} \bar{\lambda}^{(p)}\right) \cdot\right\}
\end{aligned}
$$

Furthermore, if

then

$$
X(\xi)=\psi \lambda
$$

Finally, if

$$
\left.\begin{array}{l}
\overline{\bar{X}}=\left(\bar{\psi} \bar{\lambda}^{\prime}=\alpha^{2} X(t-r)+\beta^{2} X(t+r),\right. \\
\hat{X}=\left(\bar{\psi} \hat{\lambda}^{\prime}=\alpha^{2} X(t-r)-\beta^{2} X(t+r) .\right.
\end{array}\right\}
$$

$$
Y^{\prime}(\xi)=X(\xi),
$$

then we write

$$
\left.\begin{array}{l}
\overline{\bar{Y}}=\alpha^{2} \int_{-\infty}^{t-r} X(\xi) d \xi+\beta^{2} \int_{\infty}^{t+r} X(\xi) d \xi, \\
\hat{Y}=\alpha^{2} \int_{-\infty}^{t-r} X(\xi) d \xi-\beta^{2} \int_{\infty}^{t+r} X(\xi) d \xi .
\end{array}\right\}
$$

It is worth noting that

$$
\frac{\partial \overline{\bar{X}}}{\partial r}=-\frac{\partial \hat{\hat{X}}}{\partial t}, \frac{\partial \hat{\hat{X}}}{\partial r}=-\frac{\partial \overline{\bar{X}}}{\partial t} ; \frac{\partial \overline{\bar{Y}}}{\partial r}=-\frac{\partial \hat{\hat{Y}}}{\partial t}, \frac{\partial \hat{\hat{Y}}}{\partial r}=-\frac{\partial \overline{\bar{Y}}}{\partial t} .
$$

\section{References}

Bonnor, W. B.: Phil. Trans. Roy. Soc. A, 251, 233 (1959).

EDdington, A. S.: The mathematical theory of relativity. Cambridge: Cambridge University Press 1924.

MoLLer, C.: The theory of relativity. Oxford: Oxford University Press 1962.

RotenBeRG, M. A.: Gravitational and electromagnetic waves in general relativity. London University Ph. D. thesis 1964.

— Proc. Roy. Soc. A, 293, 408 (1966). 